\title{
Calculating the Surface Topography of Integrated Circuit Wafers from SEM Images
}

\author{
Timothy P Ellison and Christopher J Taylor
}

\author{
Wolfson Image Analysis Unit \\ Department of Medical BioPhysics \\ University of Manchester \\ Manchester M19 3PT
}

The ability to make three-dimensional measurements of surface topography is important to the control of quality in the fabrication of IC wafers. We describe a technique using shape from shading to produce a dense depth map from ICs imaged in the scanning electron microscope. The method minimises error terms to ensure the resultant surface is everywhere continuous and accurately explains the observed image intensities. We describe how this method is extended when stereo images are available and how the extra information in stereocould be used to derive properties of the reflectance function.

A great deal of effort is expended by the manufacturers of integrated circuits (ICs) in judging the quality and acceptability of device interconnect metallization on integrated circuit wafers or dice. Typically they are interested in measuring parameters such as the height of tracks, passivation steps, contact windows and cross-overs of metallization layers relative to the substrate, the depth of pits in the substrate and the thickness of the glassivation layer. Due to the size of the structures involved, components are normally tested non-destructively using a scanning electron microscope (SEM). It is possible to extract some of the required quantitative information relatively easily from SEM images but heights relative to the substrate are difficult to measure. We are attempting to produce accurate measurements of three-dimensional topography from stereo images taken by SEM. An important characteristic of the problem is the variability in material properties and in imaging conditions and the consequent difficulty in modelling the imaging process.

The SEM produces images that are highly magnified representations of the sample, with surface shading similar to that observed in scenes illuminated by directional light (Figure 1). As a method of extracting three-dimensional information from such images, shape from shading [ 1,2$]$ offers the derivation of a dense depth map, which is particularly useful when trying to make volumetric measurements. Other methods commonly adopted for the extraction of three-dimensional information rely on being able to match features in a stereo pair of images (the correspondence problem). Although it is possible to obtain stereo pairs of SEM images, either by tilting the microscope stage or by deflecting the electron beam, the surfaces of interest in this application are sufficiently featureless to make the approach unattractive.

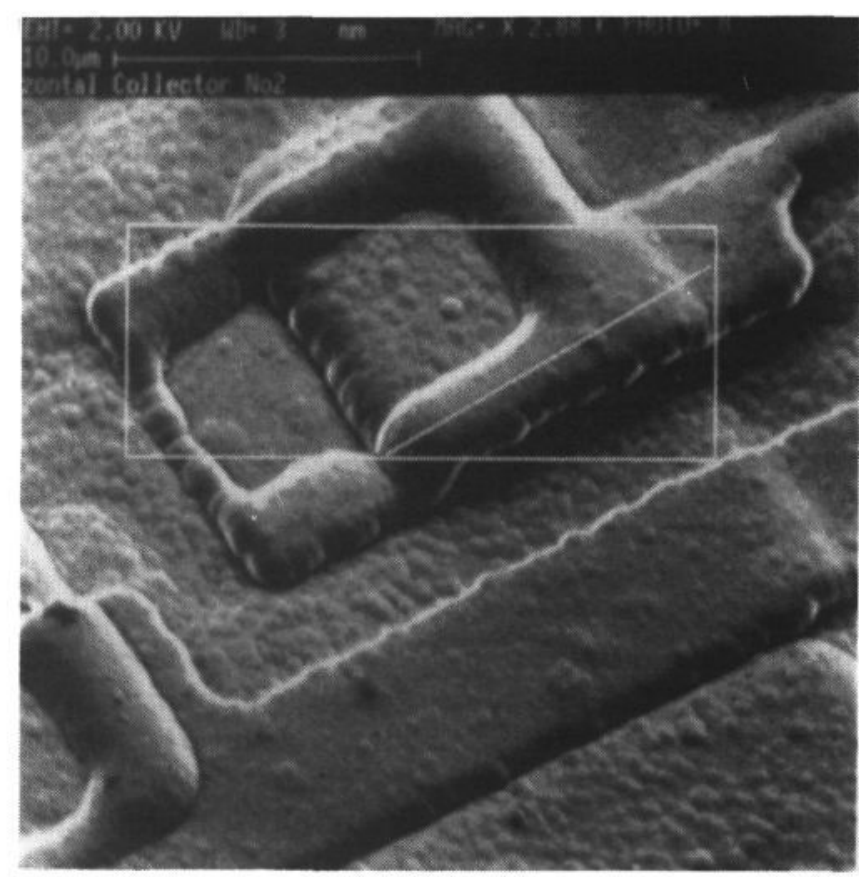

Figure 1. SEM image of an integrated circuit

This paper describes our approach to recovering height information from SEM images of ICs. Our first objective has been to exploit fully the information present in a single image. This involves making assumptions about the relationship between surface orientation and observed intensity, though it is possible to obtain an approximation to this reflectance function by making use of calibration objects. The iterative scheme we have adopted converges rapidly to to a reasonable estimate of the height map which it then adjusts directly, to achieve a least-square explanation of the observed image intensities. In order to achieve this we have developed a simple four-pass method of generating a height map from possibly inconsistent maps of surface gradient. Our second objective has been to exploit the additional information available from a stereo pair of images, using binocular shape from shading. The scheme developed for the monocular case has been extended to deal with stereo by casting the left and right image observations into a Cyclopean co-ordinate frame in which a single height map is constructed. This process requires an initial estimate of the height map, which we obtain using the monocular method. The additional information available in the binocularcase can potentially provide more accurate estimates of surface orientation 
and can resolve ambiguities present in the monocular case. We believe there is also the potential to recover information about the reflectance function though this cannot yet be demonstrated.

\section{PREVIOUS WORK}

In general, existing algorithms for extracting the shape of an object using shape from shading rely on knowing the reflectance properties of the object $a$ priori (but see [ 3 ]) expressed as a reflectance function or map. Such a function makes explicit the relationship between surface orientation and brightness, encoding information about the surface reflectance properties and light-source distribution. Most existing methods tend to make the simplifying assumption that the object exhibits Lambertian properties. This does not hold for SEM images.

The problem of obtaining the shape of an object from its shading pattern is fundamentally difficult because there are two degrees of freedom in surface orientation at each picture point but only one brightness measurement, thus we have one equation in two unknowns at each point in the image. The problem becomes tractable if a number of simplifying assumptions are made concerning the object being reconstructed and the lighting and viewing conditions under which the images were taken. Typical assumptions include the image being acquired under orthographic projection and that the underlying surface is everywhere continuous. Some methods have tended to impose unrealistic constraints on the resulting surface assuming, for example, that it is smooth [4], locally spherical [ 5 ] or hyperbolic [ 6 ]; these lead to inaccurate reconstructions of the surface. In particular shape from shading methods that solely penalise departure from smoothness will cause any non-planar surface to incur a penalty and thus tend to produce results that are flatter than the actual surface. More recently the notion of the resultant surface being integrable ${ }^{\dagger}$ has become accepted as a sufficient condition [ 8 ]. Most methods work directly with surface gradients, then recover height in a separate step [ 4 ] though methods that recover height directly have been reported recently [ 9,2$]$.

Although most papers on shape from shading only address the monocular case $[4,10,2]$ there has been some work published on obtaining shape from shading using stereo images, although they have tended to be concerned with augmenting the sparse height information available by matching stereo pairs $[11,12]$, by using the extra information available as shading to "fill in the gaps" of feature based stereo - requiring the correspondence problem to be solved.

\section{MONOCULAR SHAPE FROM SHADING}

We have derived an iterative scheme for solving the shape from shading problem whilst enforcing the integrability of the recovered surface by working directly with the height map. The method requires a good initial estimate of the height map, which we obtain using a rapidly converging but less accurate technique.

\section{Iterative Recovery of Height Map}

We use the calculus of variations [15] to derive Euler equations that provide the necessary conditions for the minimisation of several cost functions. Given the image $E(x, y)$ and a reflectance function $R(p, q)$, where $p$ and $q$ are the surface gradient in $x$ and $y$ respectively. We are interested in finding the surface $z(x, y)$ which minimises,

$$
\iint_{\Omega}(E(x, y)-R(p, q))^{2} \mathrm{~d} x \mathrm{~d} y
$$

the error, integrated over the image, between the observed intensities and those predicted using the reflectance map. We could minimise this integral in $p$ and $q$ but the result would not necessarily correspond to a continuous surface. Thus we need to find the best-fit surface $z$ which has partial derivatives $z_{x}$ and $z_{y}$ that are closest to matching the computed components of the gradient $(p$ and $q$ ) by minimising

$$
\iint_{\Omega}\left(z_{x}-p\right)^{2}+\left(z_{y}-q\right)^{2} \mathrm{~d} x \mathrm{~d} y
$$

Combining this integrability constraint with the intensity constraint, we obtain

$$
\iint_{\Omega}(E(x, y)-R(p, q))^{2}+\mu\left(\left(z_{x}-p\right)^{2}+\left(z_{y}-q\right)^{2}\right) \partial x \partial y
$$

where $\mu$ is a Lagrangian multiplier. Finding the minimum of this integral for values of $p$ and $q$ is a simple calculus problem (not involving the calculus of variations) since there are no partial derivatives of $p$ and $q$ in the function. Differentiating with respect to $p$ and $q$ and setting equal to zero results in

$$
\begin{aligned}
& p=z_{x}+\frac{1}{\mu}(E(x, y)-R(p, q)) \cdot \frac{\partial}{\partial p} R(p, q) \\
& q=z_{y}+\frac{1}{\mu}(E(x, y)-R(p, q)) \cdot \frac{\partial}{\partial q} R(p, q)
\end{aligned}
$$

To find the minimum with respect to $z$ we see that $z$ does not appear directly in $(E(x, y)-R(p, q))$, so we just need to minimise

$$
\iint_{\Omega}\left(z_{x}-p\right)^{2}+\left(z_{y}-q\right)^{2} \partial x \partial y
$$

This expression is a functional of the form $\iint \mathrm{F}\left(z, z_{x}, z_{y}\right) \mathrm{d} x \mathrm{~d} y$, and therefore has a minimum that satisfies the corresponding Euler equation,

$$
\mathrm{F}_{z}-\frac{\partial}{\partial x} \mathrm{~F}_{z_{x}}-\frac{\partial}{\partial y} \mathrm{~F}_{z_{y}}=0
$$

where

$$
\mathrm{F}=\left(z_{x}-p\right)^{2}+\left(z_{y}-q\right)^{2}
$$

hence,

$$
\frac{\partial}{\partial x}\left(z_{x}-p\right)+\frac{\partial}{\partial y}\left(z_{y}-q\right)=0
$$

is a necessary condition for a minimum in the functional. Rearranging we get

$$
\nabla^{2} z=\frac{\partial p}{\partial x}+\frac{\partial q}{\partial y}
$$


where

$$
\nabla^{2}=\frac{\partial^{2}}{\partial x^{2}}+\frac{\partial^{2}}{\partial y^{2}}
$$

We can to derive an iterative scheme to solve equation (3), by using finite-difference methods to approximate the derivatives. The discrete approximation to $\nabla^{2}$ (the Laplacian operator) is

$$
\nabla^{2} f \approx \frac{1}{\epsilon^{2}}\left(\left(f_{i, j+1}+f_{i+1, j}+f_{i, j-1}+f_{i-1, j}\right)-4 f_{i, j}\right)
$$

thus,

$$
\nabla^{2} f \approx \frac{4}{\epsilon^{2}}\left(\bar{f}_{i, j}-f_{i, j}\right)
$$

where $\epsilon$ is the inter-pixel spacing, and the local average $\bar{f}_{i, j}$ is given by

$$
\bar{f}_{i, j}=\frac{1}{4}\left(f_{i, j+1}+f_{i+1, j}+f_{i, j-1}+f_{i-1, j}\right)
$$

We can thus rewrite equation (3) in its discrete form as

$$
\frac{4}{\epsilon^{2}}\left(\bar{z}_{i, j}-z_{i, j}\right)=p_{x}+q_{y}
$$

Making $z_{i, j}$ the subject of the formula results in the iterative scheme,

$$
z_{i, j}^{k+1}=z_{i, j}^{k}-\frac{\epsilon^{2}}{4}\left(h_{i, j}^{k}+v_{i, j}^{k}\right)
$$

where

$$
\bar{z}_{i, j}=\frac{1}{4}\left(z_{i, j+1}+z_{i+1, j}+z_{i, j-1}+z_{i-1, j}\right)
$$

and

$$
h_{i, j}=\frac{1}{2}\left(p_{i+1, j}-p_{i-1, j}\right) \text { and } v_{i, j}=\frac{1}{2}\left(q_{i, j+1}-q_{i, j-1}\right)
$$

are the discrete approximations to $\epsilon p_{x}$ and $\epsilon q_{y}$.

Similarly, given $z(x, y)$, an approximation to the surface, we can change the gradients in $x$ and $y$ to minimise any difference between the observed intensities and those predicted by the current height map, by expressing equations (1) and (2) in iterative form

$$
p^{k+1}=z_{x}+\frac{1}{\mu}(E(x, y)-R(p, q)) \frac{\partial R}{\partial p}
$$

and

$$
q^{k+1}=z_{y}+\frac{1}{\mu}(E(x, y)-R(p, q)) \frac{\partial R}{\partial q}
$$

where all values on the right hand side are taken from the $\mathbf{k}^{\text {th }}$ iteration. This iterative scheme will converge to the correct solution, if one exists (ie. if there is a $E=R(p, q)$ ). On non-ideal data however, it will (in general) not reach a stable solution unless it has a good approximation to the height map from which to start. In addition, the convergence is slow since the expression that modifies the height map is dependent on the second derivative of the surface, and the feed back from the height map to the gradient maps does not constrain the gradients to describe physical surfaces.

Horn's approach is to introduce a steadily reducing smoothness term [ 14 ] which encourages the solution to converge towards a coherent surface. This does not, however, address the problem of the slow convergence of the height map. Since we are interested in using the height map at an early stage for computing the disparity in binocular images we have also considered other schemes.

\section{Obtaining an Initial Height Map}

We are interested then in finding a method for obtaining a good approximation to the underlying surface from which to begin using the exact shape from shading scheme described above. Earlier, Horn described a scheme using an integrability constraint in the gradient maps that was capable of converging rapidly to an answer near the correct solution even when given a poor initial approximation to the surface [ 2 ].

The integrability constraint enforced in the previous scheme ensures that the reconstructed surface is twice differentiable everywhere (written $C^{2}$ ). If the surface, $z$, is $\mathrm{C}^{2}$ then the order of differentiation in the two dimensions over which it is defined makes no difference to the result, ie.,

$$
\frac{\partial^{2} z}{\partial x \partial y}=\frac{\partial^{2} z}{\partial y \partial x}
$$

Rather than enforcing integrability, we can measure the departure from this condition as,

$$
\iint_{\Omega}\left(p_{y}-q_{x}\right)^{2} \mathrm{~d} x \mathrm{~d} y
$$

combining this with the intensity constraint we get,

$$
\iint_{\Omega}\left((E(x, y)-R(p, q))^{2}+\lambda\left(p_{y}-q_{x}\right)^{2}\right) \mathrm{d} x \mathrm{~d} y
$$

Now, since this functional is in the form,

$$
\mathrm{I}(p, q)=\iint_{\Omega} \mathrm{F}\left(x, y, p, q, p_{x}, p_{y}, q_{x}, q_{y}\right) \mathrm{d} x \mathrm{~d} y
$$

its extrema can be expressed as the solution of the corresponding Euler equations,

$$
\begin{aligned}
& \mathrm{F}_{p}-\frac{\partial}{\partial x} \mathrm{~F}_{p_{x}}-\frac{\partial}{\partial y} \mathrm{~F}_{p_{y}}=0 \\
& \mathrm{~F}_{q}-\frac{\partial}{\partial x} \mathrm{~F}_{q_{x}}-\frac{\partial}{\partial y} \mathrm{~F}_{q_{y}}=0
\end{aligned}
$$

Substituting the above functional for $\mathrm{F}$ and substituting into the Euler equations gives

$$
\begin{gathered}
-2(E(x, y)-R(p, q)) \frac{\partial}{\partial p} R(p, q)-\frac{\partial}{\partial y}\left(2 \lambda\left(p_{y}-q_{x}\right)\right)=0 \\
-2(E(x, y)-R(p, q)) \frac{\partial}{\partial q} R(p, q)-\frac{\partial}{\partial x}\left(-2 \lambda\left(p_{y}-q_{x}\right)\right)=0
\end{gathered}
$$

which can be simplified to read

$$
\begin{aligned}
& (E-R) R_{p}+\lambda\left(p_{y y}-q_{x y}\right)=0 \\
& (E-R) R_{q}+\lambda\left(q_{x x}-p_{y x}\right)=0
\end{aligned}
$$

where again subscripts denote the relevant partial differentiation and the appropriate arguments $(x, y)$ and $(p, q)$ are assumed for $E$ and $R$.

We now wish to devise an iterative scheme to solve this pair of equations. We can use finite-difference methods to approximate the differentials,

$$
\frac{\partial^{2} f}{\partial y^{2}} \approx \frac{1}{\epsilon^{2}}\left(\left(f_{i, j+1}+f_{i, j-1}\right)-2 f_{i, j}\right)
$$


Which we can rewrite as

$$
\frac{\partial^{2} f}{\partial y^{2}} \approx \frac{2}{\epsilon^{2}}\left(\tilde{f}_{i, j}-f_{i, j}\right)
$$

where $\bar{f}_{i, j}=\frac{1}{2}\left(f_{i, j+1}+f_{i, j-1}\right)$

is the vertical local average of $f_{i, j}$.

Now, taking equation (7) as the example, rearranging and substituting $p_{y y}$ gives,

$$
q_{x y}-\left(\frac{2}{\epsilon^{2}}(\bar{p}-p)\right)=\frac{1}{\lambda}(E-R) R_{p}
$$

and therefore

$$
p=\bar{p}-\frac{\epsilon^{2}}{2} q_{x y}+\frac{\epsilon^{2}}{2 \lambda}(E-R) R_{p}
$$

Using the discrete approximation,

$$
\frac{\partial^{2} q}{\partial x \partial y} \approx \frac{1}{4 \epsilon^{2}}\left(q_{i+1, j+1}+q_{i-1, j-1}-q_{i-1, j+1}-q_{i+1, j-1}\right)
$$

We have the iterative scheme,

$$
p^{k+1}=\vec{p}^{k}-\frac{1}{2} \tilde{q}^{k}+\frac{\epsilon^{2}}{2 \lambda}\left(E-R\left(p^{k}, q^{k}\right)\right) R_{p}\left(p^{k}, q^{k}\right)
$$

where

$$
\begin{gathered}
\bar{p}_{i, j}=\frac{1}{2}\left(p_{i, j+1}+p_{i, j-1}\right) \text { and } \\
\tilde{q}_{i, j}=\frac{1}{4}\left(q_{i+1, j+1}+q_{i-1, j-1}-q_{i-1, j+1}-q_{i+1, j-1}\right)
\end{gathered}
$$

are the vertical average of $p$ and an estimate of the cross derivative (times $\epsilon^{2}$ ) of $q$ respectively.

Equation (8) yields a similar iterative scheme for $q$.

This method does not enforce integrability of the resultant surface, but merely treats it as an error term to be minimised together with the intensity difference. Its ability to start from a poor approximation to the surface and its rapid convergence make it suitable for deriving an initial surface from which to start iterating the exact scheme.

\section{Full Monocular Method}

We have combined the two methods described above to produce a shape from shading scheme which converges rapidly to an accurate estimate of height starting from a set of $p, q$ and $z$ maps that do not represent a surface near the correct answer.

We use the method of equation (9) to build $p$ and $q$ maps in which departures from integrability are penalised. Once this method has converged we cast the gradient values onto a coherent physical surface by scanning through the gradient maps from each corner of the reconstructed region, at each point producing a surface height that best explains the local gradients whilst imposing integrability. Using the notation $p$ and $q$ as before, the height at a point $(x, y)$ is given in terms of the scan direction by,

$$
\begin{aligned}
z_{m n}(x, y)= & \left(\frac{z_{m n}(x-m, y)+m p(x-m, y)}{8}+\right. \\
\left.\frac{z_{m n}(x, y-n)+n q(x, y-n)}{8}\right) & \\
z(x, y)= & z_{11}(x, y)+z_{-11}(x, y)+z_{1-1}(x, y)+z_{-1-1}(x, y)
\end{aligned}
$$

where $m= \pm 1, n= \pm 1$ denote the direction of the scan in $x$ and $y$ respectively.

Once the initial height map has been constructed we continue the method of equation (9), except that now we recalculate the $p$ and $q$ maps from $z$ at every iteration. This will not converge to the correct solution but ensures that the solution maintains convergence during the initial stages of introducing feedback from the height map. Once stable convergence has been re-established the method of equations (4), (5) and (6) is applied. In practice, we do not make this final change instantaneously but rather take a weighted combination of the two methods to achieve a graceful transition.

\section{BINOCULAR SHAPE FROM SHADING}

We obtain shape from binocular shading from stereo images by building the height and orientation maps with reference to a global co-ordinate system (GCS) based upon the Cyclopean view. The monocular shape from shading algorithm is extended to use the shading information available in both images. The extension of the algorithm involves transforming image points from the co-ordinate systems of the left and right eye views (LCS and $\mathrm{RCS}$ respectively) to the GCS, using information from the height map. The nature of the iteration is such that the correspondence improves as the height map approaches the correct solution. The error function is easily extended to take account of the extra information available.

If we make the simplifying assumption of oblique projection, then the mapping from GCS to LCS and RCS becomes a simple rotation. Using no subscript to denote the GCS, the subscript " $L$ " for the LCS and the subscript " $R$ " for the RCS and extending the scheme described above,

$$
\begin{aligned}
2 p=z_{x} & +\frac{1}{\mu}\left(E_{L}-R\left(p_{L}, q_{L}\right)\right) \frac{\partial R\left(p_{L}, q_{L}\right)}{\partial p}+ \\
z_{x} & +\frac{1}{\mu}\left(E_{R}-R\left(p_{R}, q_{R}\right)\right) \frac{\partial R\left(p_{R}, q_{R}\right)}{\partial p}
\end{aligned}
$$

A similar equation holds for $q$; the equation in $z$ remains unchanged.

As with the exact monocular method the scheme requires a reasonably good starting approximation. We obtain this by computing an initial monocular solution.

\section{RESULTS}

\section{Monocular Method}

The monocular method has been applied to both synthesised images and real SEM images. In both cases measurements made from calibration objects, viewed in the SEM,were used to estimate the reflectance function. Cylinders and spheres were used as calibration objects since surface orientation could be inferred very simply 


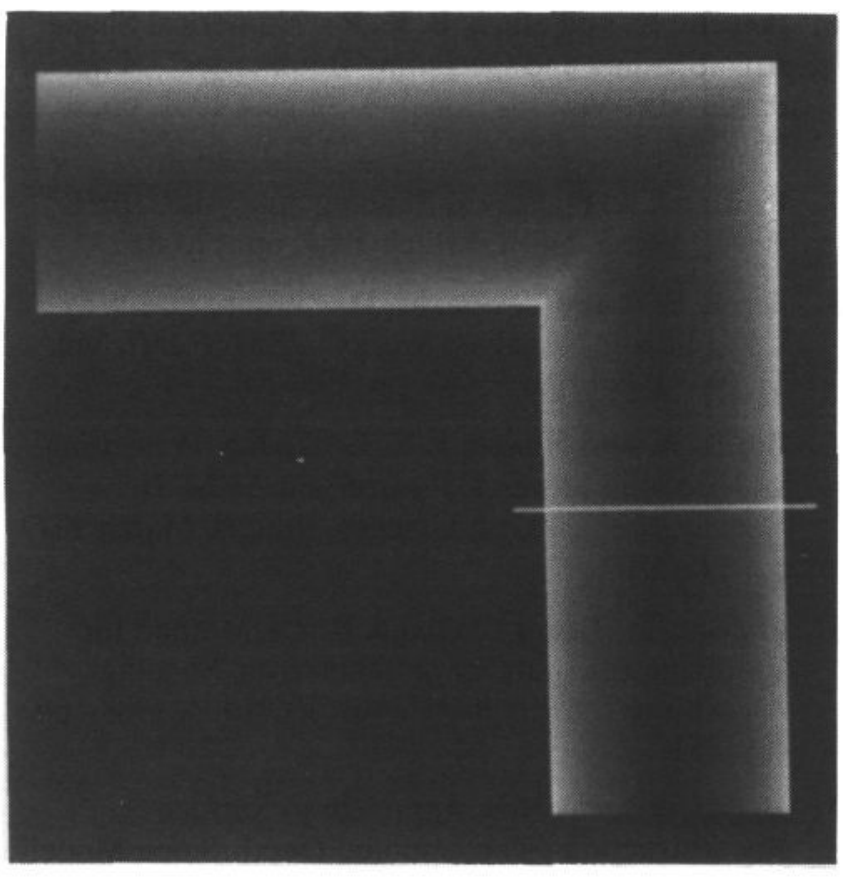

Figure 2. Synthesized SEM image of a cylinder

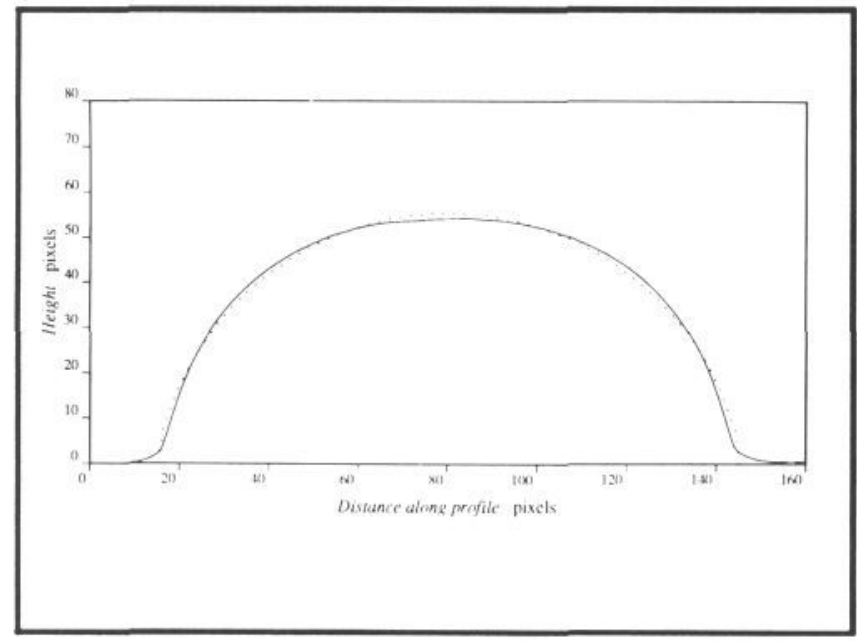

Figure 3. Height profile computed from Figure 2

from radial position in the projected image. The effects of noise were minimised by fitting polynomial surfaces through the measured data. Synthetic images of cylinders were generated from the reflectance function and used to test the methodology. Figure 2 shows one such test image of a 64 pixel radius cylinder. Figure 3 shows the calculated height profile, at the position indicated in Figure 2, after a total of 120 iterations (Phase $1=60$, Phase $2=60$ ), starting from a plane surface. The solution was stable. The dashed line shows a least-square circle fitted to the data, indicating a calculated diameter of 65 pixels.

Figure 4 shows a height profile of the surface reconstructed from the IC image in Figure 1 using the same procedure as above; again the solution was stable. No absolute height data is available for comparison but the profile appears qualitatively correct and quantitatively plausible.

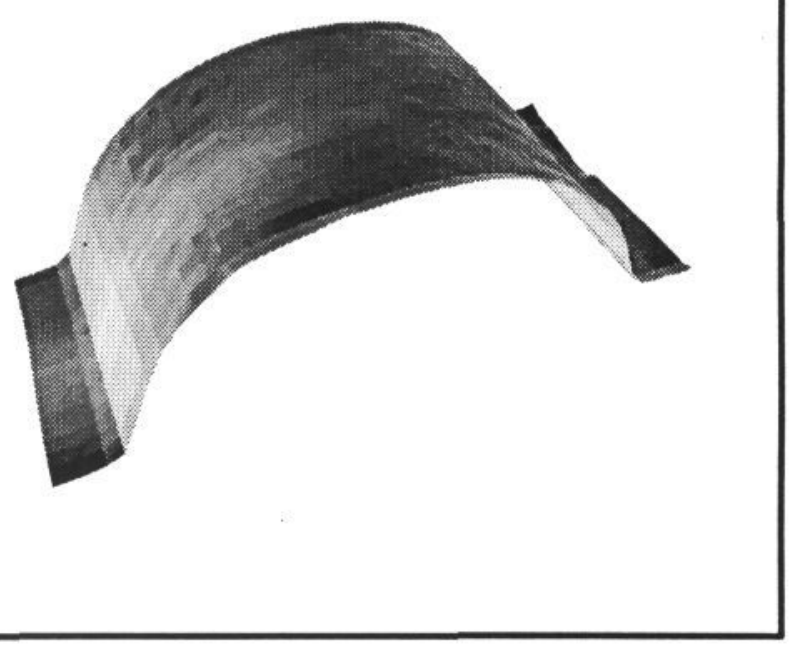

Figure 5. Rendered surface reconstructed by binocular method

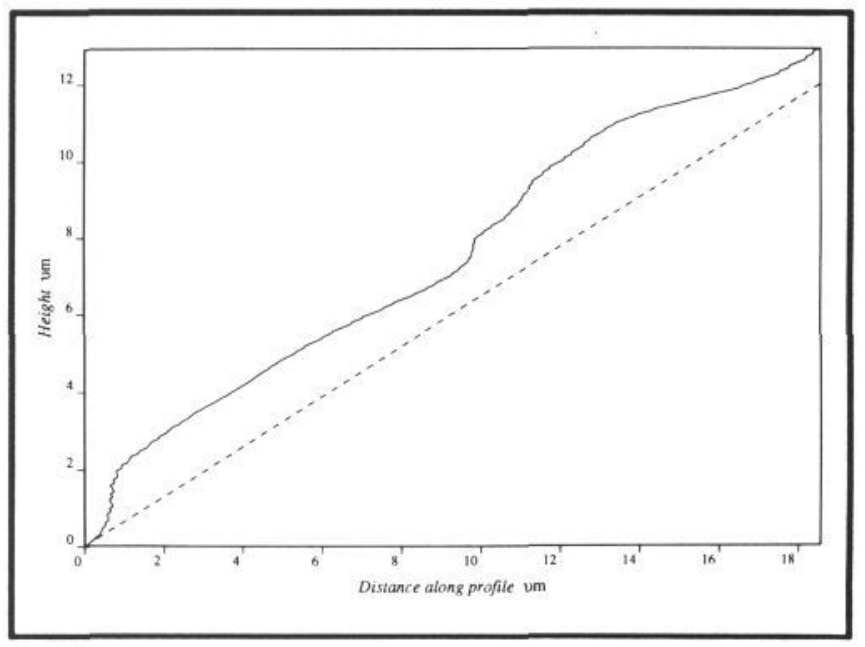

Figure 4. Height profile computed from Figure 1

\section{Binocular Method}

The binocular method has only been applied to synthetic data so far but we hope to present results obtained for real SEM images, at BMVC 90 . Synthetic images of cylinders were generated for $\pm 3^{\circ}$ beam tilt. The monocular method was used to provide an initial estimate of the height map as described above. The binocular method converged to a stable solution after about 20 iterations. A rendered illustration of the calculated surface is shown in Figure 5.

\section{DISCUSSION}

We have shown that it is possible to reconstruct plausible height maps from monocular SEM images of ICs, using a method that converges in tens of iterations starting from a planar surface. We have shown, using synthetic data, that the method can be successfully extended to deal with 
binocular images. We are currently undertaking an experimental determination of the accuracy of both methods, using real SEM images of calibration objects; the results with synthetic images indicate that, given a good estimate of the reflectance map, it is possible to achieve accuracies of a few per cent in height measurement. We have pointed out that it is not necessarily practical to obtain a prior estimate of the reflectance map and we intend to further investigate the possibility of using the redundancy present in binocular shaded images to recover the reflectance map on-line. Once a reasonably stable solution for the height map has been reached, using an initial approximation to the reflectance map, we intend to modify the iterative scheme to allow $\mathrm{R}(p, q)$ to be updated to improve agreement between observed and predicted intensities. Stability will be maintained by applying a very strong smoothness constraint in the reflectance map.

Further improvements in speed may be obtainable using multi-grid methods though previous attempts to apply these techniques to shape from shading have achieved only limited success [ 13 ].

Although many of the surfaces of interest in this application are smoothly varying there are also localised topographical features which could be used for feature-based stereo and it seems likely that a practical scheme for extracting height from SEM images would also need to incorporate such methods.

\section{ACKNOWLEDGEMENTS}

Tim Ellison holds a Total Technology studentship funded by SERC and Leica Cambridge Ltd.

\section{REFERENCES}

1. Horn, B. K. P. Robot Vision MIT Press, 1986

2. Horn, B. K. P. and Brooks, M. J. Shape from Shading MIT Press, 1989

3. Brooks, M. J. and Horn, B. K. P. "Shape and Source from Shading" Proc. of the Intern. Joint Conf. on Artificial Intelligence, Los Angeles, California, 18-23 August 1985, pp. 932-936
4. Ikeuchi, K. and Horn, B. K. P. "Numerical Shape from Shading and Occluding Boundaries" Artificial Intelligence, Vol. 17, Nos. 1-3, August 1981, pp. 141-184

5. Pentland, A. P. "Local Shading Analysis" IEEE PAMI, Vol. 6, No. 2, March 1984, pp. 170-187

6. Ferrie, F. P. and Levine, M. D. "Where and Why Local Shading Analysis Works" IEEE PAMI, Vol. 11, No. 2, February 1989, pp. 198-206

7. Boyde, A. and Howell, P. G. T. "Taking, Presenting and Treating Stereo Data from the SEM. II.” Scanning/1977, Part I, Chicago, Illinois, March 1977 pp. $571-580$

8. Frankot, R. T. and Chellapa, R. "A Method for Enforcing Integrability in Shape from Shading Algorithms" IEEE PAMI, Vol. 10, No. 4, 1988, pp. $439-451$

9. Harris, J. J. "A New Approach to Surface Reconstruction : The Coupled Depth/Slope Model" ICCV, London, June 8-11,1987, pp. 277-283

10 . Horn, B. K. P. and Brooks, M. J. "The Variation Approach to Shape from Shading" MIT AI Lab. Memo 813, March 1985

11 . Ikeuchi, K. "Constructing a Depth map from Images” AI Memo No. 744, MIT AI Lab., Cambridge, MA, 1983

12 . Grimson, W. E. L. "Binocular Shading and Visual Surface Reconstruction" Computer Vision, Graphics and Image Processing, Vol. 28, 1984, pp 19-43

13 . Ron, G. and Peleg, S. "Multiresolution Shape from Shading", IEEE PAMI, 1989, pp.350-355

14 . Horn, B. K. P. "Height and Gradient from Shading" MIT AI Laboratory Memo AI-M-1105, May 1989

15 . Courant, R. and Hilbert, D. Methods of Mathematical Physics Volume 1 Interscience Publishers Ltd., London, 1953

16. Lee, D. "A Provably Convergent Algorithm for Shape from Shading" Proceedings CVPR, June 1988, Ann Arbour, MI, pp. 478-485, 1988 